\title{
ASYMPTOTIC BEHAVIOR OF ORTHOGONAL POLYNOMIALS CORRESPONDING TO A MEASURE WITH INFINITE DISCRETE PART OFF AN ARC
}

\author{
R. KHALDI and R. BENZINE
}

(Received 23 October 2000)

\begin{abstract}
We study the asymptotic behavior of orthogonal polynomials. The measure is concentrated on a complex rectifiable arc and has an infinity of masses in the region exterior to the arc.
\end{abstract}

2000 Mathematics Subject Classification. 42C05, 30E15, 30E10.

1. Introduction. Kaliaguine has studied in [3] the asymptotic behavior of orthogonal polynomials associated to a measure of the type $\sigma_{l}=\alpha+\gamma_{l}$, where $\alpha$ is concentrated on a complex rectifiable arc $E$ and is absolutely continuous with respect to the Lebesgue measure $|d \xi|$ on the arc, and $\gamma_{l}$ is a finite discrete measure with masses $A_{k}$ at the points $z_{k} \in \operatorname{Ext}(E), k=1,2, \ldots, l$, that is, $\gamma_{l}=\sum_{k=1}^{l} A_{k} \delta_{z_{k}}, A_{k}>0$, where $\delta_{z_{k}}$ being the Dirac measure at the points $z_{k}$. In this paper, we generalize the previous study, when $\sigma=\alpha+\gamma$, where $\alpha$ possess the same properties as in [3] and $\gamma$ is concentrated on an infinite discrete part $\left\{z_{k}\right\}_{k=1}^{\infty} \in \operatorname{Ext}(E), \gamma=\sum_{k=1}^{\infty} A_{k \delta_{z_{k}}}$. The masses $\left\{A_{k}\right\}_{k=1}^{\infty}$ satisfy

$$
A_{k}>0, \quad \sum_{k=1}^{\infty} A_{k}<\infty .
$$

We note that the cases of a closed curve and a circle studied in $[4,5]$ are different from the case of an arc with respect to the asymptotics of orthogonal polynomials.

2. The space $H^{2}(\Omega, \rho)$. Suppose that $E$ is a rectifiable arc in the complex plane, $\Omega=\operatorname{Ext}(E), G=\{w \in C /|w|>1\}(\infty \in \Omega, \infty \in G)$, and $1 / C(E)=\lim _{z \rightarrow \infty}(\Phi(z) / z)>0$, where $\Phi: \Omega \rightarrow G$ is the conformal mapping. We denote by $\Psi$ the inverse of $\Phi$.

Let $\rho(\xi)$ be an integrable nonnegative function on $E$. If the weight function $\rho(\xi)$ satisfies the Szegö condition

$$
\int_{E} \log (\rho(\xi))\left|\Phi^{\prime}(\zeta)\right||d \xi|>-\infty .
$$

Then one can construct the so-called Szegö function $D(z)$ associated with the domain $\Omega$ and the weight function $\rho(\xi)$ with the following properties.

$D(z)$ is analytic in $\Omega ; D(z) \neq 0$ in $\Omega ; D(\infty)>0 ; D(z)$ has boundary values on both sides of $E$ (a.e.) and $\left|D_{ \pm}\right|^{-2}\left|\Phi_{ \pm}^{\prime}\right|=\rho(\xi)$ (a.e. on $E$ ).

Let $f(z)$ be an analytic function in $\Omega$, we say that $f(z) \in H^{2}(\Omega, \rho)$ if and only if $f(\Psi(w)) / D(\Psi(w)) \in H^{2}(G)$, and for a function $F$ analytic in $G, F \in H^{2}(G)$ if and only if 
$F(1 / w) \in H^{2}(D) ; w \in D ; D=\{z \in C /|z|<1\}$. The space $H^{2}(D)$ is well known (see [6]). Any function from $H^{2}(\Omega, \rho)$ has boundary values $f_{+}, f_{-}$on both sides of $E, f_{+}, f_{-} \in L^{2}(\rho)$. We define the norm in Hardy space by

$$
\|f\|_{H^{2}(\Omega, \rho)}=\oint_{E}|f(\xi)|^{2} \rho(\xi)|d \xi| .
$$

Here, we take the integral on both sides of $E$.

3. Extremal properties of the orthogonal polynomials. We denote by $P_{n}$ the set of polynomials of degree almost $n$. Define $\mu(\rho), \mu^{*}(\rho), m_{n}(\rho), m_{n}\left(\sigma_{l}\right)$, and $m_{n}(\sigma)$ as the extremal values of the following problems:

$$
\begin{aligned}
& \mu(\rho)=\inf \left\{\|\varphi\|_{H^{2}(\Omega, \rho)}^{2}: \varphi \in H^{2}(\Omega, \rho), \varphi(\infty)=1\right\}, \\
& \mu^{*}(\rho)=\inf \left\{\|\varphi\|_{H^{2}(\Omega, \rho)}^{2}: \varphi \in H^{2}(\Omega, \rho), \varphi(\infty)=1, \varphi\left(z_{k}\right)=0, k=1,2, \ldots\right\}, \\
& m_{n}(\rho)=\min \left\{\int_{E}\left|Q_{n}(\xi)\right|^{2} \rho(\xi)|d \xi|, Q_{n}(z)=z^{n}+\cdots\right\}, \\
& m_{n}\left(\sigma_{l}\right)=\min \left\{\int_{E}\left|Q_{n}(\xi)\right|^{2} \rho(\xi)|d \xi|+\sum_{k=1}^{l} A_{k}\left|Q_{n}\left(z_{k}\right)\right|^{2}, Q_{n}(z)=z^{n}+\cdots\right\}, \\
& m_{n}(\sigma)=\min \left\{\int_{E}\left|Q_{n}(\xi)\right|^{2} \rho(\xi)|d \xi|+\sum_{k=1}^{\infty} A_{k}\left|Q_{n}\left(z_{k}\right)\right|^{2}, Q_{n}(z)=z^{n}+\cdots\right\} .
\end{aligned}
$$

We denote, respectively, by $\varphi^{*}$ and $\psi^{*}$ the extremal functions of the problems (3.1) and (3.2). We denote by $\left\{T_{n}^{l}(z)\right\}$ and $\left\{T_{n}(z)\right\}$ the systems of the monic orthogonal polynomials, respectively, associated to the measures $\sigma_{l}$ and $\sigma$, that is,

$$
\begin{gathered}
T_{n}^{l}(z)=z^{n}+\cdots, \\
\int_{E} T_{n}^{l}(\xi) \bar{\xi}^{p} \rho(\xi)|d \xi|+\sum_{k=1}^{l} A_{k} T_{n}^{l}\left(z_{k}\right) \bar{\xi}_{k}^{p}=0 ; \quad p=0,1,2, \ldots, n-1, \\
T_{n}(z)=z^{n}+\cdots, \\
\int_{E} T_{n}(\xi) \bar{\xi}^{p} \rho(\xi)|d \xi|+\sum_{k=1}^{\infty} A_{k} T_{n}\left(z_{k}\right) \bar{\xi}_{k}^{p}=0 ; \quad p=0,1,2, \ldots, n-1 .
\end{gathered}
$$

It is easy to see that the polynomials $\left\{T_{n}^{l}(z)\right\}$ and $\left\{T_{n}(z)\right\}$ are, respectively, the optimal solutions of the extremal problems (3.4) and (3.5).

LEMMA 3.1. Let $\varphi \in H^{2}(\Omega, \rho)$ such that $\varphi(\infty)=1$ and $\varphi\left(z_{k}\right)=0, k=1,2, \ldots$, and let

$$
B(z)=\prod_{k=1}^{\infty} \frac{\Phi(z)-\Phi\left(z_{k}\right)}{\Phi(z) \overline{\Phi\left(z_{k}\right)}-1} \frac{\left|\Phi\left(z_{k}\right)\right|^{2}}{\Phi\left(z_{k}\right)}
$$

be the Blashke product, then 
(1) $B \in H^{2}(\Omega, \rho) ; B(\infty)=1 ;\left|B_{ \pm}(\xi)\right|=\prod_{k=1}^{\infty}\left|\Phi\left(z_{k}\right)\right|$ (a.e. on $E$ ),

(2) $\varphi / B \in H^{2}(\Omega, \rho)$ and $(\varphi / B)(\infty)=1$.

The proof is the same as that of Lemma 3.1 given in [1].

LEMMA 3.2. The extremal functions $\varphi^{*}$ and $\psi^{*}$ are connected by

$$
\psi^{*}=B(z) \cdot \varphi^{*}, \quad \mu^{*}(\rho)=\left(\prod_{k=1}^{\infty}\left|\Phi\left(z_{k}\right)\right|\right)^{2} \mu(\rho) .
$$

The proof is the same as that of a closed curve given in [2, Lemma 4.2]. We replace the finite Blashke product by the infinite product $B$ and using its properties announced by Lemma 3.1.

\section{Main results}

Definition 4.1. The measure $\sigma=\alpha+\gamma$ belongs to the class $A$ (and we write $\sigma \in A$ ), if the absolutely continuous part $\alpha$ and the discrete part of $\sigma$ satisfy (in addition to conditions (1.1) and (2.1))

$$
\left(\sum_{k=1}^{\infty}\left|\Phi\left(z_{k}\right)\right|-1\right)<\infty .
$$

An $\operatorname{arc} E$ is from $C^{\alpha+}$ class if $E$ is rectifiable and its coordinates are $\alpha$-times differentiable, with $\alpha$ th derivatives satisfying a Lipschitz condition positive exponent.

THEOREM 4.2. Let $\sigma$ be a measure, $\sigma=\alpha+\gamma$, such that $\sigma \in A$. Then

$$
\lim _{l \rightarrow \infty} m_{n}\left(\sigma_{l}\right)=m_{n}(\sigma)
$$

Proof. The extremal property of $T_{n}(z)$ implies that

$$
m_{n}\left(\sigma_{l}\right) \leq \int_{E}\left|T_{n}(\xi)\right|^{2} \rho(\xi)|d \xi|+\sum_{k=1}^{l} A_{k}\left|T_{n}\left(z_{k}\right)\right|^{2} \leq m_{n}(\sigma),
$$

then

$$
m_{n}\left(\sigma_{l}\right) \leq m_{n}(\sigma)
$$

On the other hand, the extremal property of $T_{n}(z)$ implies that

$$
\begin{aligned}
m_{n}(\sigma) & \leq \int_{E}\left|T_{n}^{l}(\xi)\right|^{2} \rho(\xi)|d \xi|+\sum_{k=1}^{\infty} A_{k}\left|T_{n}^{l}\left(z_{k}\right)\right|^{2} \\
& =m_{n}\left(\sigma_{l}\right)+\sum_{k=l+1}^{\infty} A_{k}\left|T_{n}^{l}\left(z_{k}\right)\right|^{2} .
\end{aligned}
$$

According to the reproducing property of the kernel function $K_{n}(\xi, z)$ (see [7]), and $T_{n}^{l}(z) \in P_{n}$, we have

$$
T_{n}^{l}\left(z_{k}\right)=\int_{E} T_{n}^{l}(\zeta) \overline{K_{n}\left(\xi, z_{k}\right)} \rho(\xi)|d \xi| .
$$


The Scharwz inequality and the fact that $|\Phi(\xi)|=1$ for $\xi \in E$ and $K_{n}\left(z, z_{k}\right) \in P_{n}$ imply

$$
\begin{aligned}
\left|T_{n}^{l}\left(z_{k}\right)\right|^{2} & \leq \int_{E}\left|T_{n}^{l}(\xi)\right|^{2} \rho(\xi)|d \xi| \cdot \int_{E}\left|K_{n}\left(\xi, z_{k}\right)\right|^{2} \rho(\xi)|d \xi| \\
& \leq m_{n}\left(\sigma_{l}\right) \cdot K_{n}\left(z_{k}, z_{k}\right) .
\end{aligned}
$$

The inequalities (1.1), (4.5), and (4.7) imply

$$
\begin{aligned}
m_{n}(\sigma) & \leq m_{n}\left(\sigma_{l}\right)+\sum_{k=l+1}^{\infty} A_{k} m_{n}\left(\sigma_{l}\right) K_{n}\left(z_{k}, z_{k}\right) \\
& \leq m_{n}\left(\sigma_{l}\right)\left[1+\sup _{k \geq l+1} K_{n}\left(z_{k}, z_{k}\right) \sum_{k=l+1}^{\infty} A_{k}\right],
\end{aligned}
$$

so we have

$$
\frac{m_{n}(\sigma)}{m_{n}\left(\sigma_{l}\right)} \leq 1+\delta_{l}, \quad \text { where } \delta_{l} \longrightarrow 0, l \rightarrow \infty
$$

Using (4.4) and (4.9), we obtain

$$
m_{n}(\sigma) \leq \liminf _{l \rightarrow \infty} m_{n}\left(\sigma_{l}\right) \leq \limsup _{l \rightarrow \infty} m_{n}\left(\sigma_{l}\right) \leq m_{n}(\sigma), \quad \forall n,
$$

this implies that

$$
\lim _{l \rightarrow \infty} m_{n}\left(\sigma_{l}\right)=m_{n}(\sigma), \quad \forall n
$$

THEOREM 4.3. Let $\sigma$ be a measure, $\sigma=\alpha+\gamma$, such that $\sigma \in A$ and

$$
\frac{m_{n}\left(\sigma_{l}\right)}{m_{n}(\rho)} \leq\left(\prod_{k=1}^{l}\left|\Phi\left(z_{k}\right)\right|\right)^{2}, \quad \forall n, \forall l .
$$

Suppose that $E \in C^{2+}$. Then we have

$$
\begin{gathered}
\lim _{n \rightarrow \infty} \frac{m_{n}(\sigma)}{C(E)^{2 n}}=\mu^{*}(\rho), \\
\int_{E}\left|C(E)^{-n} T_{n}(\xi)-H_{n}(\xi)\right|^{2} \rho(\xi)|d \xi| \rightarrow 0, \\
T_{n}(z)=C(E)^{n} \Phi^{n}(z)\left[\psi^{*}(z)+\epsilon_{n}(z)\right],
\end{gathered}
$$

where $H_{n}(\xi)=\Phi_{+}^{n}(\xi) \psi_{+}^{*}(\xi)+\Phi_{-}^{n}(\xi) \psi_{-}^{*}(\xi), \epsilon_{n} \rightarrow 0$ uniformly on the compact subsets of $\Omega$.

Proof. By passing to the limit when $l$ tends to infinity and using Theorem 4.2 and (4.12), we obtain

$$
\frac{m_{n}(\sigma)}{C(E)^{2 n}} \leq\left(\prod_{k=1}^{\infty}\left|\Phi\left(z_{k}\right)\right|\right)^{2} \frac{m_{n}(\rho)}{C(E)^{2 n}}
$$

This implies that

$$
\limsup _{n \rightarrow \infty} \frac{m_{n}(\sigma)}{C(E)^{2 n}} \leq\left(\prod_{k=1}^{\infty}\left|\Phi\left(z_{k}\right)\right|\right)^{2} \mu(\rho)=\mu^{*}(\rho)
$$

(see Lemma 3.2). 
The extremal property of the polynomials $T_{n}(z)$ and the fact that $|\Phi(\xi)|=1$, for $\xi \in E$ imply (see [2] for details)

$$
\frac{2 m_{n}(\sigma)}{C(E)^{2 n}}=\left\|\frac{T_{n}}{[C(E) \Phi]^{n}}\right\|_{H^{2}(\Omega, \rho)}^{2}+2 \sum_{k=1}^{\infty} A_{k}\left|\frac{T_{n}\left(z_{k}\right)}{\left[C(E) \Phi\left(z_{k}\right)\right]^{n}}\right|\left|\Phi\left(z_{k}\right)\right|^{2 n},
$$

so

$$
\left\|\frac{T_{n}}{[C(E) \Phi]^{n}}\right\|_{H^{2}(\Omega, \rho)}^{2} \leq \frac{2 m_{n}(\sigma)}{C(E)^{2 n}} .
$$

Equations (4.15) and (4.17) imply that

$$
\limsup _{n \rightarrow \infty}\left\|\frac{T_{n}}{[C(E) \Phi]^{n}}\right\|_{H^{2}(\Omega, \rho)}^{2} \leq 2 \mu^{*}(\rho) .
$$

Now we take the integral

$$
\begin{aligned}
I_{n}=\int_{E} \mid & C(E)^{-n} T_{n}(\xi)-\left.H_{n}(\xi)\right|^{2} \rho(\xi)|d \xi| \\
=\int_{E} \mid & \left(\frac{1}{2} C(E)^{-n} T_{n}(\xi)-\Phi_{+}^{n}(\xi) \psi_{+}^{*}(\xi)\right) \\
& +\left.\left(\frac{1}{2} C(E)^{-n} T_{n}(\xi)-\Phi_{-}^{n}(\xi) \psi_{-}^{*}(\xi)\right)\right|^{2} \rho(\xi)|d \xi|,
\end{aligned}
$$

by the triangular inequality, we have

$$
\begin{aligned}
I_{n}^{1 / 2} \leq & \left(\int_{E}\left|\frac{1}{2} C(E)^{-n} T_{n}(\xi)-\Phi_{+}^{n}(\xi) \psi_{+}^{*}(\xi)\right|^{2} \rho(\xi)|d \xi|\right)^{1 / 2} \\
& +\left(\int_{E}\left|\frac{1}{2} C(E)^{-n} T_{n}(\xi)-\Phi_{-}^{n}(\xi) \psi_{-}^{*}(\xi)\right|^{2} \rho(\xi)|d \xi|\right)^{1 / 2} \\
\leq & 2\left(\oint_{E}\left|\frac{1}{2} C(E)^{-n} T_{n}(\xi)-\Phi^{n}(\xi) \psi^{*}(\xi)\right|^{2} \rho(\xi)|d \xi|\right)^{1 / 2} .
\end{aligned}
$$

Then we deduce that

$$
I_{n} \leq 4\left\|\frac{1}{2} \frac{T_{n}}{[C(E) \Phi]^{n}}-\psi^{*}\right\|_{H^{2}(\Omega, \rho)}^{2} .
$$

By using the parallelogram rule in $H^{2}(\Omega, \rho)$, we have

$$
I_{n} \leq 4\left[2\left\|\frac{1}{2} \frac{T_{n}}{C(E)^{n}}\right\|_{H^{2}(\Omega, \rho)}^{2}+2\left\|\psi^{*}\right\|_{H^{2}(\Omega, \rho)}^{2}-\left\|\frac{1}{2} \frac{T_{n}}{C(E)^{n}}+\psi^{*}\right\|_{H^{2}(\Omega, \rho)}^{2}\right],
$$

so

$$
\limsup _{n \rightarrow \infty} I_{n} \leq 4\left[\mu^{*}(\rho)+2 \mu^{*}(\rho)-\frac{9}{4} \frac{4}{3} \mu^{*}(\rho)\right]=0,
$$

where we have used the fact that $\liminf _{n \rightarrow \infty}\left\|g_{n}\right\|_{H^{2}(\Omega, \rho)}^{2} \geq 2 \mu^{*}(\rho) \geq(4 / 3) \mu^{*}(\rho)$, since the function $g_{n}(z)=(2 / 3)\left((1 / 2) T_{n}(z) / C(E)^{n}+\psi^{*}(z)\right) \in H^{2}(\Omega, \rho), g_{n}(\infty)=1$, and $g_{n}\left(z_{k}\right) \rightarrow 0, n \rightarrow \infty$. This yields

$$
0 \leq \liminf _{n \rightarrow \infty} I_{n} \leq \limsup _{n \rightarrow \infty} I_{n} \leq 0,
$$


finally,

$$
\lim _{n \rightarrow \infty} I_{n}=0 .
$$

For the asymptotics in the region exterior to the $\operatorname{arc} E$ we need the Szegö reproducing kernel function $K(\xi, z)$ (see [8, page 173]) and the fact that $T_{n}(z) / C(E)^{n} \Phi^{n}(z) \in$ $H^{2}(\Omega, \rho)$ for all $z \in \Omega$, then

$$
\begin{aligned}
\frac{T_{n}(z)}{C(E)^{n} \Phi^{n}(z)}= & \oint_{E} \frac{T_{n}(\xi)}{C(E)^{n} \Phi^{n}(\xi)} \overline{K(\xi, z)} \rho(\xi)|d \xi| \\
= & \int_{E} C^{-n} T_{n}(\xi)\left\{\Phi_{+}^{-n}(\xi) \overline{K_{+}(\xi, z)}+\Phi_{-}^{-n}(\xi) \overline{K_{-}(\xi, z)}\right\} \rho(\xi)|d \xi| \\
= & \int_{E}\left\{C^{-n} T_{n}(\xi)-H_{n}(\xi)\right\}\left\{\Phi_{+}^{-n}(\xi) \overline{K_{+}(\xi, z)}+\Phi_{-}^{-n}(\xi) \overline{K_{-}(\xi, z)}\right\} \rho(\xi)|d \xi| \\
& +\int_{E} H_{n}(\xi)\left\{\Phi_{+}^{-n}(\xi) \overline{K_{+}(\xi, z)}+\Phi_{-}^{-n}(\xi) \overline{K_{-}(\xi, z)}\right\} \rho(\xi)|d \xi| .
\end{aligned}
$$

The first integral approaches 0 as $n \rightarrow \infty$ (part 2 of Theorem 4.3), the second one may be transformed into the form

$$
\begin{aligned}
\int_{E}\left\{\Phi_{+}^{n}(\xi) \psi_{+}^{*}(\xi)+\Phi_{-}^{n}(\xi) \psi_{-}^{*}(\xi)\right\}\left\{\Phi_{+}^{-n}(\xi) \overline{K_{+}(\xi, z)}+\Phi_{-}^{-n}(\xi) \overline{K_{-}(\xi, z)}\right\} \rho(\xi)|d \xi| \\
=\oint_{E} \psi^{*}(\xi) \overline{K(\xi, z)} \rho(\xi)|d \xi| \\
\quad+\int_{E}\left\{\Phi_{+}^{n}(\xi) \psi_{+}^{*}(\xi) \Phi_{-}^{-n}(\xi) \overline{K_{-}(\xi, z)}+\psi_{-}^{*}(\xi) \Phi_{+}^{-n}(\xi) \Phi_{-}^{n}(\xi) \overline{K_{+}(\xi, z)}\right\} \rho(\xi)|d \xi| \\
=\psi^{*}(z)+\lambda_{n},
\end{aligned}
$$

where $\lambda_{n} \rightarrow 0$ (coefficients of an integrable function). This proves part 3.

REMARK 4.4. It is not difficult to find families of points $\left\{A_{k}\right\}_{k=1}^{\infty}$ and $\left\{z_{k}\right\}_{k=1}^{\infty}$ satisfying condition (4.12). For example if $E=[-1,+1]$, then

$$
\Phi(z)=z+\sqrt{z^{2}-1} \quad\left(\left|z+\sqrt{z^{2}-1}\right|>1\right) .
$$

We can take $z_{k}$ such that

$$
\Phi\left(z_{k}\right)=1+\frac{1}{k^{2}}, \quad A_{k}=\frac{1}{2^{k}} .
$$

As weight function we take

$$
\rho(\xi)=\left(1-\xi^{2}\right)^{-1 / 2}
$$

\section{REFERENCES}

[1] R. Benzine, Asymptotic behavior of orthogonal polynomials corresponding to a measure with infinite discrete part off a curve, J. Approx. Theory 89 (1997), no. 2, 257-265. MR 98g:42036. Zbl 882.42014.

[2] V. Kaliaguine and R. Benzine, Sur la formule asymptotique des polynômes orthogonaux associés à une mesure concentrée sur un contour plus une partie discrète finie, Bull. Soc. Math. Belg. Sér. B 41 (1989), no. 1, 29-46 (French). MR 91f:42025. Zbl 683.42027. 
[3] V. A. Kaliaguine, A note on the asymptotics of orthogonal polynomials on a complex arc: the case of a measure with a discrete part, J. Approx. Theory 80 (1995), no. 1, 138-145. MR 95k:42042. Zbl 831.42015.

[4] R. Khaldi and R. Benzine, Asymptotic behavior of a class of orthogonal polynomials on the circle: case of measures with an infinite discrete part, no. 411, Publications du Laboratoire d'Analyse Numerique et d'Optimisation, Lille, 2000.

[5] _ On a generalization of an asymptotic formula of orthogonal polynomials, Int. J. Appl. Math. 4 (2000), no. 3, 261-274.

[6] W. Rudin, Real and Complex Analysis, McGraw-Hill, New York, 1966. MR 35\#1420. Zbl 142.01701.

[7] G. Szegö, Orthogonal Polynomials, 4th ed., American Mathematical Society, Colloquium Publications, vol. 23, American Mathematical Society, Rhode Island, 1975. MR 51\#8724.

[8] H. Widom, Extremal polynomials associated with a system of curves in the complex plane, Advances in Math. 3 (1969), 127-232. MR 39\#418. Zbl 183.07503.

R. Khaldi: DePARTMEnT of MATHEMAtics, University of ANNABA, B.P. 12 AnNABA, ALGERIA

R. Benzine: Department of Mathematics, University of AnnABA, B.P. 12 AnNabA, ALGERIA

E-mail address: benzine@ifrance.com 


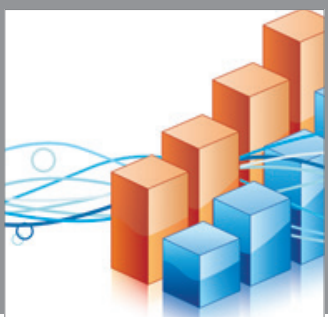

Advances in

Operations Research

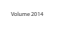

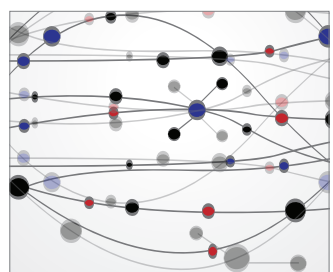

\section{The Scientific} World Journal
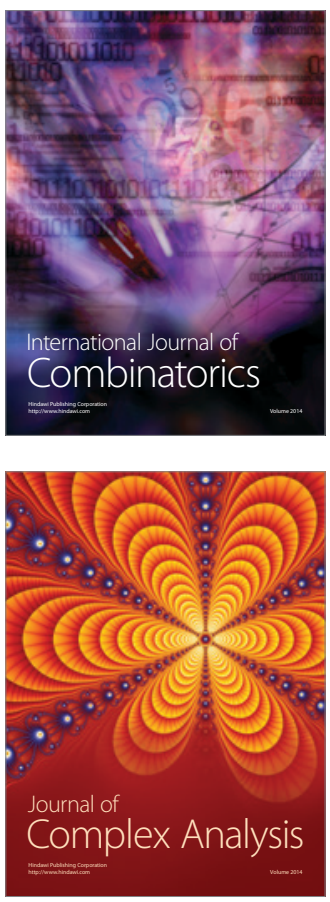

International Journal of

Mathematics and

Mathematical

Sciences
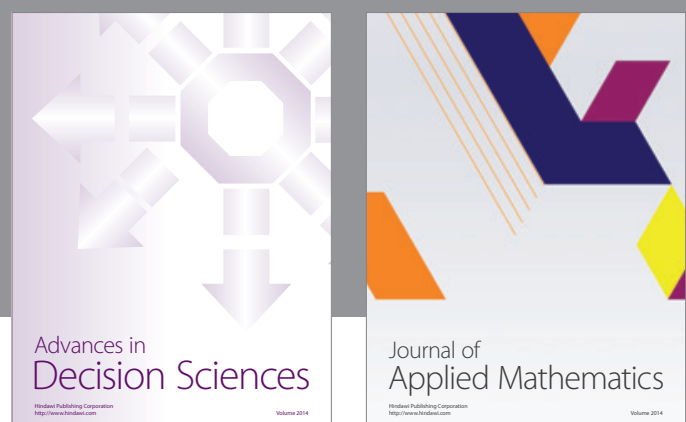

Journal of

Applied Mathematics
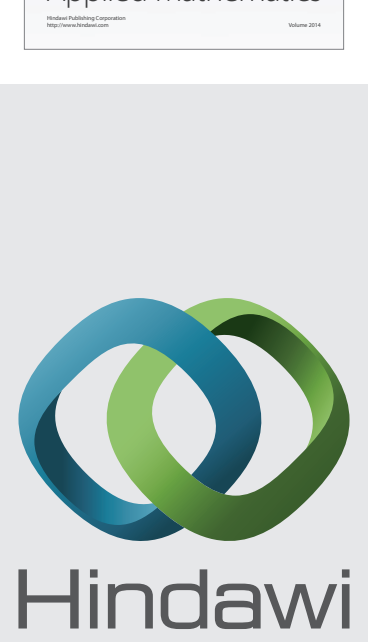

Submit your manuscripts at http://www.hindawi.com
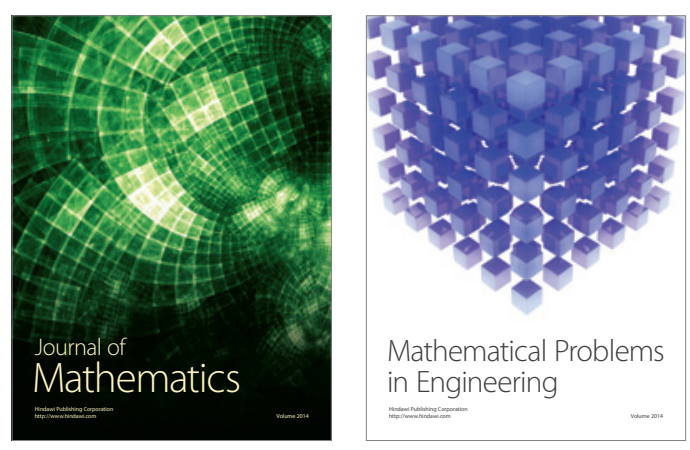

Mathematical Problems in Engineering
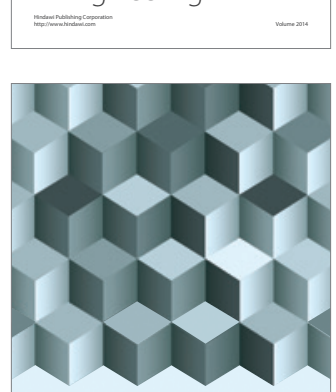

Journal of

Function Spaces
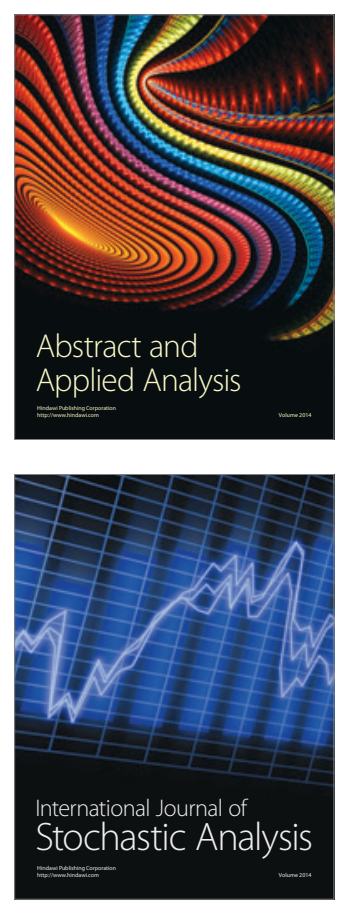

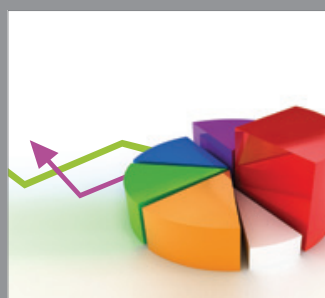

ournal of

Probability and Statistics

Promensencen
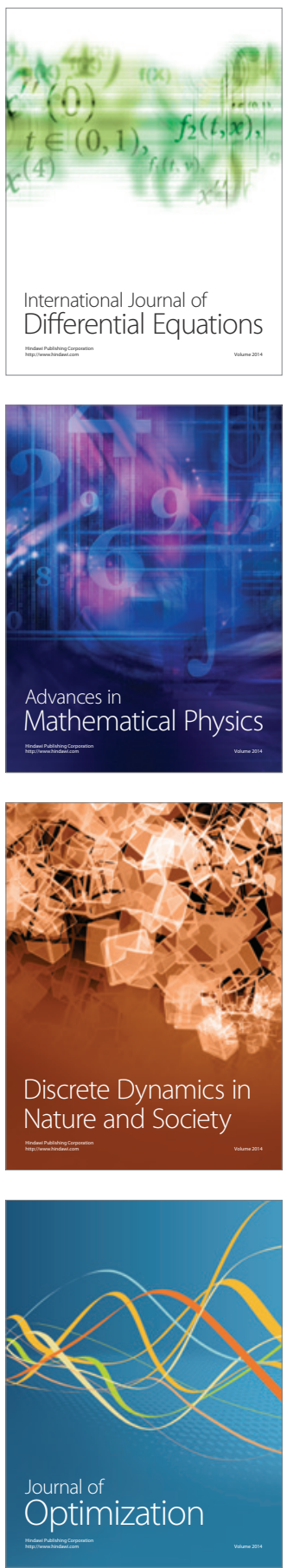